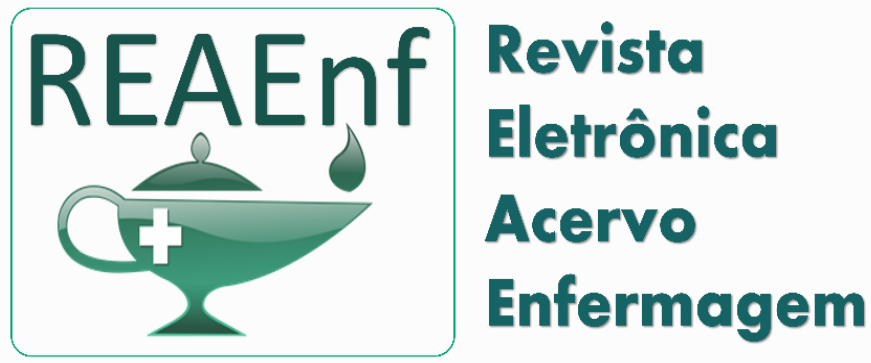

ARTIGO ORIGINAL

Recebido em: 8/2020

Aceito em: 9/2020

Publicado em: 2/2021

\title{
Rastreamento dos riscos para desenvolvimento de diabetes mellitus tipo 2 em duas populações distintas
}

\author{
Risk tracking for the development of diabetes mellitus type 2 in two different populations \\ Seguimiento de riesgos para el desarrollo de diabetes mellitus tipo 2 en dos poblaciones \\ diferentes
}

Arimatéia Portela de Azevedo ${ }^{1 *}$, Erika Brasil Fernandes ${ }^{1}$, Bruno Pinto Metteles ${ }^{1}$, Patrícia Brasil Augusto de Souza ${ }^{1}$, Cleise Pereira Simplício ${ }^{1}$, Bruno Alves Almeida ${ }^{1}$, Adriana Rodrigues Batista da Gama $^{1}$, Greyciane Coêlho de Souza Rodrigues ${ }^{1}$, Samuel Aquino de Araújo ${ }^{1}$, Antônia Evanilda Alves do Nascimento ${ }^{1}$.

\begin{abstract}
Resumo: Realizado rastreamento para descobrir fatores de risco para desenvolver diabetes mellitus tipo 2 em servidores da saúde de um hospital referência em infectologia e moradores de um bairro classe baixa na periferia de Manaus. Estudo prospectivo, descritiva com abordagem quantitativa onde foi utilizado um questionário chamado Finnish Diabetes Risk Score (FINDRISC), que pontua os fatores de risco e os classifica em escore da projeção da possibilidade para desenvolver o diabetes mellitus tipo 2 em dez anos. Participaram da pesquisa 120 pessoas com idade média de 30 anos sendo 40 comunitários e 80 servidores da saúde. Destes, $43 \%$ encontravam-se com risco para desenvolver diabetes mellitus tipo 2 (DM2) em dez anos. Entre as variáveis que apontavam os riscos, foi encontrado aumento significativo de indivíduos com circunferência abdominal acima dos parâmetros considerados normais $(84,0 \%)$, e sedentarismo (80\%), que consumiam frituras diariamente e salgados ou carnes gordas (75\%), e também fator hereditariedade (90\%). Ar, luz solar, dieta balanceada e a pratica de exercício físicos apresentam-se necessária para o controlo da doença.
\end{abstract}

Palavras-chave: Diabetes mellitus tipo 2, Inquéritos epidemiológicos, Serviços preventivos de saúde.

\begin{abstract}
Screening was carried out to discover risk factors for developing type 2 diabetes mellitus in health workers of a reference hospital in infectology and residents of a low-class neighborhood on the outskirts of Manaus. Prospective, descriptive study with a quantitative approach where a questionnaire called Finnish Diabetes Risk Score (FINDRISC) was used, which scores the risk factors and classifies them in the score of the projection of the possibility to develop type 2 diabetes mellitus in ten years. 120 people participated in the research, with an average age of 30 years, 40 of whom were community members and 80 were health workers. Of these, $43 \%$ were at risk for developing type 2 diabetes mellitus (DM2) in ten years. Among the variables that pointed out the risks, a significant increase was found in individuals with abdominal circumference above the parameters considered normal (84.0\%), and sedentary lifestyle (80\%), who consumed fried foods daily and salty or fatty meats $(75 \%)$, and also heredity factor $(90 \%)$. Air, sunlight, a balanced diet and physical exercise are necessary to control the disease.
\end{abstract}

Keywords: Type 2 diabetes mellitus, Health surveys, Preventive health services.

${ }^{1}$ Fundação de Medicina Tropical Dr. Heitor Vieira Dourado (FMT/HVD), Manaus - AM.

*E-mail: arimateia@fmt.am.gov.br 
Resumen: Se realizó un cribado para descubrir factores de riesgo para desarrollar diabetes mellitus tipo 2 en trabajadores de salud de un hospital de referencia en infectología y residentes de un barrio de clase baja en las afueras de Manaus. Estudio prospectivo, descriptivo con enfoque cuantitativo donde se utilizó un cuestionario denominado Finnish Diabetes Risk Score (FINDRISC), que puntúa los factores de riesgo y los clasifica en el score de la proyección de posibilidad de desarrollar diabetes mellitus tipo 2 en diez años. En la investigación participaron 120 personas, con una edad promedio de 30 años, 40 de los cuales eran miembros de la comunidad y 80 eran trabajadores de la salud. De estos, el $43 \%$ tenían riesgo de desarrollar diabetes mellitus tipo 2 (DM2) en diez años. Entre las variables que señalaron los riesgos, se encontró un aumento significativo en individuos con circunferencia abdominal por encima de los parámetros considerados normales $(84,0 \%)$ y sedentarios $(80 \%)$, que consumían diariamente frituras y carnes saladas 0 grasas $(75 \%)$, y también factor de herencia $(90 \%)$. El aire, la luz solar, una dieta equilibrada y el ejercicio físico son necesarios para controlar la enfermedad.

Palabras clave: Diabetes mellitus tipo 2, Encuestas de salud, Servicios de salud preventiva.

\section{INTRODUÇÃO}

Dados epidemiológicos sobre a Diabetes no Brasil mostram que o crescente número de idosos chega a $4 \%$ ao ano, no período de 2012 a 2022, e a população idosa com 60 anos ou mais chegará a 41,5 milhões em 2030. O Estado brasileiro com a menor prevalência de diabéticos é Brasília (5,2\%) e a maior prevalência está em São Paulo, mais especificamente no Município de São Carlos (13,5\%) (SOCIEDADE BRASILEIRA DE DIABETES, 2019).

A Diabetes Mellitus (DM) representa uma pandemia global, na qual se avalia que 300 milhões de pessoas, nos próximos 20 anos, apresentarão a doença, é dividida principalmente em dois tipos: Diabetes Mellitus tipo 1 (DM1), também chamada de diabetes juvenil ou insulinodependente, doença autoimune que atinge cerca de $10 \%$ da população de diabéticos, e Diabetes Mellitus tipo 2 (DM2), que atinge em torno de 90\% dessa população e que apresenta prejuízos na ação e produção da insulina (ARELLI B, et al., 2014).

O tipo 2 é sempre associada ao sedentarismo e a obesidade. Ela se destaca em relação às patologias consideradas graves principalmente em países em desenvolvimento como no Brasil, pela evidencia nas suas complicações com predomínio nos fatores de risco cardiovasculares (ARAÚJO LO, et al., 2015).

Essa patologia causa uma desordem metabólica de etiologia múltipla, e torna-se uma doença crônica apresentando distúrbios no metabolismo de carboidratos, proteínas e lipídeos causados pela resistência tecidual periférica ou ausência de resposta insulínica associada a uma deficiência relativa da insulina, os quais contribui para o aparecimento da hiperglicemia (ANUHARYS P, et al., 2014).

Ela causa uma condição crônica e a sua prevalência está relacionada também à idade, ao estilo de vida e estresse da vida urbana. Dados sobre prevalência em nove capitais brasileiras indicam que em média 7,6\% dos brasileiros entre 30 a 69 anos de idade apresentam DM (ANDRADE I, et al., 2014).

A Sociedade Brasileira de Diabetes (SBD), descreve que há um importante crescimento evidenciado na mortalidade relacionado a essa doença, que também tem mostrando outra importância no crescimento relacionado a outras afecções, pelos estudos feitos nos anos de 2000 a 2015 que demonstram o diabetes como causa de morte (SBD, 2019). Vale ressaltar, portanto, que a metade da população brasileira com diabetes mellitus desconhece a existência da patologia, e cerca de 1/5 dos indivíduos que estão cientes das suas condições de saúde, não procuram tratamento médico adequado, situações como esta influenciam nos indicadores das unidades básicas que as pessoas portadoras no Brasil não recebem a assistência adequada de saúde (GBD, 2017).

Em 1988 a Constituição Federal Brasileira, estabeleceu pilares para adequação de um sistema universal com objetivo democrático e humanitário, o Sistema Único de Saúde (SUS), assim adequando a atenção básica em uma realidade de descentralização e hierarquização da assistência (BRASIL, 2013).

A Estratégia de Saúde da Família (ESF) é caracterizada como linha de reconstrução de um modelo de atenção à saúde da nação brasileira, tem como finalidade garantir a qualidade, integração e eficácia no 
primeiro plano de atenção, tendo como fundamento o trabalho sob a região e a oferta de serviços, em trabalhos de promoção, proteção e reabilitação a saúde (BRASIL, 2015).

Segundo SBD, na população de baixa renda os fatores que se destacam estão interligados a alimentação errônea como consumo de ácidos graxos saturados, açúcares e refrigerantes e consumo de carboidratos complexos, com redução no consumo de frutas, verduras e legumes, o sedentarismo, histórico familiar de diabetes em parentes de 1 grau, presença de sobrepeso, obesidade e hipertensão artéria (SBD, 2019).

Mesmo assim, observa-se uma grande prevalência de diabetes na população carente. Os dados de incidências e agravos ganham respostas do sistema de saúde, esta resposta está relacionada à uma nova estrutura dos serviços de saúde e ao processo de assistência. O ideal seria uma assistência em todos os níveis de complexidade que desse suporte a essa condição e realidade de cada indivíduo e diminuísse, em longo prazo, a sua incidência assim como as atividades de prevenção e agravos e promoção de saúde voltada ao controle do diabetes (BORGES DB, et al., 2018).

Portanto, mediante tudo que foi exposto acima, o objetivo deste foi investigar, conhecer e comparar informações sobre o risco de desenvolvimento de DM2 entre servidores de um hospital referência em infectologia e moradores de um bairro periférico, classe baixa, de Manaus.

\section{MÉTODOS}

Este foi um estudo do tipo prospectivo, descritivo, com abordagem quantitativa onde a coleta de dados foi realizada, tanto na comunidade como entre os servidores da saúde que trabalhavam na Fundação de Medicina Tropical, através de um movimento promovido pela Agência Adventista de Desenvolvimento e Recursos Assistenciais (ADRA) em parceria com o serviço de educação em saúde da Fundação de Medicina Tropical.

Foi montada uma tenda de atendimento na comunidade que serviu como triagem para os atendimentos de: aferição de pressão arterial, glicemia capilar, cálculo de Índice de Massa Corpórea (IMC), medição da circunferência abdominal, mensuração da altura e aplicação de uma enquete com oito perguntas sobre o estilo de vida. Para os profissionais da saúde, o atendimento foi realizado no local de trabalho de cada participante. Cada variável do questionário é pontuada, e sua soma categoriza o participante de acordo com o grau de risco em: baixo risco, pouco elevado, alto e muito alto. Além disso, é um questionário útil para a identificação de pessoas com diabetes não diagnosticada e pré-diabetes, com $85 \%$ de precisão aqui no Brasil.

Para avaliação antropométrica, foi utilizado o resultado da divisão da grandeza do peso em quilogramas $(\mathrm{Kg})$ pela altura ao quadro em metros $(\mathrm{m})$ para mensurar o índice de massa corporal (IMC). Para aferição do peso corporal, foi usada uma balança digital de vidro com capacidade para $180 \mathrm{~kg}$ de alta precisão e divisão de 100 gramas Jasm Scales. Para o teste de glicemia capilar foi utilizado o aparelho portátil G-Tech Free 1, com tiras e lancetas estéreis e descartáveis. Os dados foram tabulados com dupla digitação em planilha do programa Microsoft $\Theta$ Excel e analisados de forma estatística através do software live $R$ versão 3.0®.5.1.

Além do questionário Finnish Diabetes Risk Score (Finrisk) também foi utilizado um questionário Socioeconômico, confeccionado pelos autores, composto de perguntas fechadas: profissão, escolaridade, turno de trabalho, setor, tempo de exercício na profissão, tempo de serviço no setor e quantidade de vínculos empregatícios (caso fosse servidor da saúde). Além de um campo para registrar a glicemia capilar, pressão arterial com data e horário. O mesmo questionário foi utilizado, tanto para servidores da saúde quanto moradores da comunidade, somente era levado em consideração, com o termo "não se aplica", algumas variáveis que não cabia para determinado grupo. As perguntas foram feitas de forma simples e com linguagem acessível aos comunitários e servidores.

Após a aplicação dos questionários foi mensurada as medidas antropométricas (peso, altura, circunferência abdominal). Ao término das medidas antropométricas, foi realizado o teste de glicemia capilar, seguindo as técnicas de punção digital até se obter 01 (uma) gota de sangue e o resultado era 
mostrado na tela do aparelho. A tira teste, a lanceta e os algodões utilizados eram descartados em recipientes adequados para descarte de material perfuro cortante. Os resultados foram registrados com a data e hora em formulário específico.

Após a aplicação dos instrumentos os mesmos eram orientados conforme a classificação de risco, sendo que os grupos risco alto e risco muito alto eram agendados para consulta com uma nutricionista ao término da entrevista. Também era entregue um panfleto com o nome do participante e o resultado da glicemia capilar com orientações de prevenção do Programa Municipal de Atenção Básica.

Após a aplicação dos instrumentos mencionados e contagem dos pontos obtidos no questionário, foi informado ao participante o resultado da seguinte maneira:

- $\quad$ < que sete pontos (Risco baixo): Mantenha suas consultas habituais.

- 7 a 11 pontos (Risco pouco elevado): Mantenha suas consultas habituais e tente modificar seu estilo de vida conforme as sugestões existentes no panfleto fornecido pelo Programa Municipal de Atenção Básica (HIPERDIA).

- $\quad 12$ a 14 pontos (Risco moderado): Mantenha suas consultas habituais e tente modificar seu estilo de vida conforme as sugestões no panfleto fornecido pelo Programa Municipal de Atenção Básica (HIPERDIA).

- 15 a 20 pontos (Risco alto): Procure orientação na Estratégia Saúde da Família (Casinha) mais próxima em até 03 (três) meses para avaliar a glicemia e as intervenções necessárias. Aquele participante do estudo que se enquadrava nesse grupo era de imediato encaminhado a uma consulta com a Nutricionista a fim de receber orientação nutricional.

- Acima de 20 pontos (Risco muito alto): Procure orientação na Estratégia Saúde da Família (Casinha) mais próxima em até 1 (um) mês para avaliar a glicemia e as intervenções necessárias. $O$ participante que se enquadrava nesse grupo era encaminhado a uma consulta com a Nutricionista.

Para a circunferência abdominal foram considerados como parâmetros normais $\geq 102 \mathrm{~cm}$ para homens e $\geq 88 \mathrm{~cm}$ para mulheres, como valores de risco DM tipo II e DCV segundo o Consenso Latino-Americano de Obesidade (1999).

O Índice de Massa Corpórea (IMC), foi calculado conforme o índice de Quetelet: divisão do Peso (P) pela altura (A) elevada ao quadrado. IMC $=\mathrm{P} / \mathrm{A}^{2}$, e os resultados conforme delimita a Organização Mundial da Saúde (2008) para peso normal $\left(18,5-24,9 \mathrm{~kg} / \mathrm{m}^{2}\right)$, baixo peso $\left(<18,5 \mathrm{~kg} / \mathrm{m}^{2}\right)$, sobrepeso $\left(25-29,9 \mathrm{~kg} / \mathrm{m}^{2}\right)$ e obeso $\left(30-\geq 40 \mathrm{~kg} / \mathrm{m}^{2}\right)$.

Não foram elegíveis para o estudo grávidas por falta de um consenso uniforme de triagem, diagnóstico e tratamento da Sociedade Brasileira de Diabetes e servidores com diagnóstico de diabetes mellitus tipo II. Também não fizeram parte da amostra participantes que não concordaram em participar da pesquisa ou e recusar a assinar o Termo de Consentimento Livre Esclarecido (TCLE). A pesquisa teve início após a aprovação pelo Comitê de Ética em Pesquisa (CEP) da Fundação de Medicina Tropical Dr. Heitor Vieira Dourado (FMT/HVD), de acordo com a resolução 466/12 do Ministério da Saúde, número do perecer 580.627, sob o CAAE 27947614.4.0000.0005.

\section{RESULTADOS E DISCUSSÃO}

Fizeram parte da pesquisa 120 pessoas com idade média de 30 anos sendo 40 participantes atendidos durante a ação de saúde na comunidade e 80 servidores da saúde com a maioria (62\%) sendo do gênero feminino. Do total, $43 \%$ encontravam-se com risco para desenvolver essa patologia. Ao avaliar os escores de riscos do questionário FINDRISC, foi constatada associação estatisticamente significante entre o risco para desenvolver DM2 e as variáveis da circunferência abdominal aumentada, ao sedentarismo, ao consumo diário de frituras, salgados ou carnes gordas e aos fatores ligados a hereditariedade.

Em relação aos servidores da saúde que participaram do estudo 47,8\% eram da área administrativa, $36,8 \%$ assistenciais e $15,8 \%$ dos laboratórios, $67,5 \%$ tinham mais de um vínculo empregatício $32,5 \%$ 
trabalhavam em plantões noturnos e 25,2\% estavam acima do peso. As resposta expressas no questionário Diabetes Risk Score mostrou que $11,25 \%$ se encontraram em alto risco e que o gênero masculino apresentou maior risco para desenvolver DM2 em dez anos e as classes de profissionais que mais apresentaram risco foram os técnicos de enfermagem (37\%) e técnicos de laboratórios com $(17,5 \%)$ que já estavam a mais de 10 anos exercendo a profissão e com mais de 2 vínculos empregatício.

Percebeu-se que, quanto mais próximo da assistência direta ao paciente, maior o número de vínculos empregatícios e tempo de serviço, maior o cansaço físico, estresse, os riscos de desenvolver a diabetes aumentavam. O sedentarismo foi o fator de risco predominante nos dois grupos. Também a hereditariedade, idade superior a 45 anos, o hábito do tabagismo e a Hipertensão Arterial auto referida em $17,5 \%$ dos participantes (Quadro 1).

Quadro 1 - Comparativo dos fatores de risco nos participantes atendidos na comunidade e nos servidores da saúde.

\begin{tabular}{|lcc|}
\hline Fatores de riscos & $\begin{array}{l}\text { Visto entre os } \\
\text { atendidos na } \\
\text { comunidade }\end{array}$ & $\begin{array}{c}\text { Visto entre os } \\
\text { servidores da } \\
\text { saúde }\end{array}$ \\
\hline Que se consideravam brancos & $45 \%$ & $56,8 \%$ \\
\hline Que se consideravam negros & $50 \%$ & $41,2 \%$ \\
\hline Que se consideravam asiáticos & $5 \%$ & $2,0 \%$ \\
\hline Com pressão alta ou toma remédios para controlá-la & $20 \%$ & $80 \%$ \\
\hline $\begin{array}{l}\text { Que tinham parentes em primeiro grau (pais ou irmão } \\
\text { e filhos) com diabetes }\end{array}$ & $35 \%$ & $65 \%$ \\
\hline $\begin{array}{l}\text { Com parentes em segundo grau (tios, tias, avós e } \\
\text { primos em primeiro grau) com diabetes? }\end{array}$ & $55 \%$ & $45 \%$ \\
\hline $\begin{array}{l}\text { Que realiza atividade física regularmente: Mais de 30 } \\
\text { minutos por dia ou mais de 4 horas por semana }\end{array}$ & $65 \%$ & $35 \%$ \\
\hline $\begin{array}{l}\text { Que realiza atividade física regularmente: Menos de } \\
\text { 30 minutos por dia ou menos de 4 horas por semana }\end{array}$ & $20 \%$ & $80 \%$ \\
\hline $\begin{array}{l}\text { Que consumia diariamente vegetais, frutas, legumes } \\
\text { ou grãos }\end{array}$ & $40 \%$ & $60 \%$ \\
\hline $\begin{array}{l}\text { Que consumia diariamente frituras, salgados ou } \\
\text { carnes gordas (incluindo frango com pele) }\end{array}$ & $75 \%$ & $25 \%$ \\
\hline Fumava de 1 a 10 cigarros por dia & $55 \%$ & $45 \%$ \\
\hline
\end{tabular}

Fonte: Azevedo AP, et al., 2020.

Em relação as informações colhidas do inquérito com os moradores da comunidade, quanto aos fatores de risco modificáveis, destacou-se o excesso de peso e obesidade. No gênero masculino $27 \%$ estavam com o IMC na faixa de sobrepeso sendo que $20,0 \%$ se declararam hipertensos. Esse dado coaduna com o que foi encontrado em outros estudos (O'DONNELL MJ, et al., 2016).

A maioria dos participantes investigados da comunidade não tinham o hábito de realizar atividade físicas e os que realizavam, praticavam menos que 30 minutos por dia. Apenas 20,0\% desses participantes estavam fora do índice de sedentarismo (Quadro 1).

Estudos indicam que esse motivo é necessário que as intervenções sejam trabalhadas no ambiente familiar para que as mesmas se tornem eficazes gerando resultados positivos (SKALAMERA J, et al., 2016).

As publicações sobre esse tema dizem que o motivo da elevação do desenvolvimento até a mortalidade diante das doenças crônicas e cardiovasculares é a falta de atividade física insuficiente, de modo que as pessoas que não praticam atividade física ativamente representam um risco de mortalidade de 20 a $30 \%$ maior que os outros riscos, quando se compara aqueles que praticam regularmente FOUAD M, et al., 2016).

Quanto à qualidade da alimentação, entre todos os investigados, $75 \%$ informaram que consumiam diariamente carnes gordas e frituras, sendo que a maioria relata hábitos alimentares não saudáveis (Gráfico 1). Esse é o segundo maior risco para desenvolvimento de diabetes. 
Um estudo demonstrou que apenas $40,0 \%$ da população adulta de menor nível socioeconômico consumiam regularmente frutas, legumes e verduras, e $80 \%$ não praticavam atividade física regularmente. O hábito de boas práticas alimentares e o incentivo à prática de exercício físico deve ser iniciado na infância, consolidados na adolescência e reforçados na fase adulta e idosa, contribuindo como fatores protetores para saúde (MAZZINI MRC, et al., 2016).

Gráfico 1 - fatores de riscos mais recorrentes encontrados nos 120 participantes dos dois grupos investigados (comunitários e servidores da saúde).

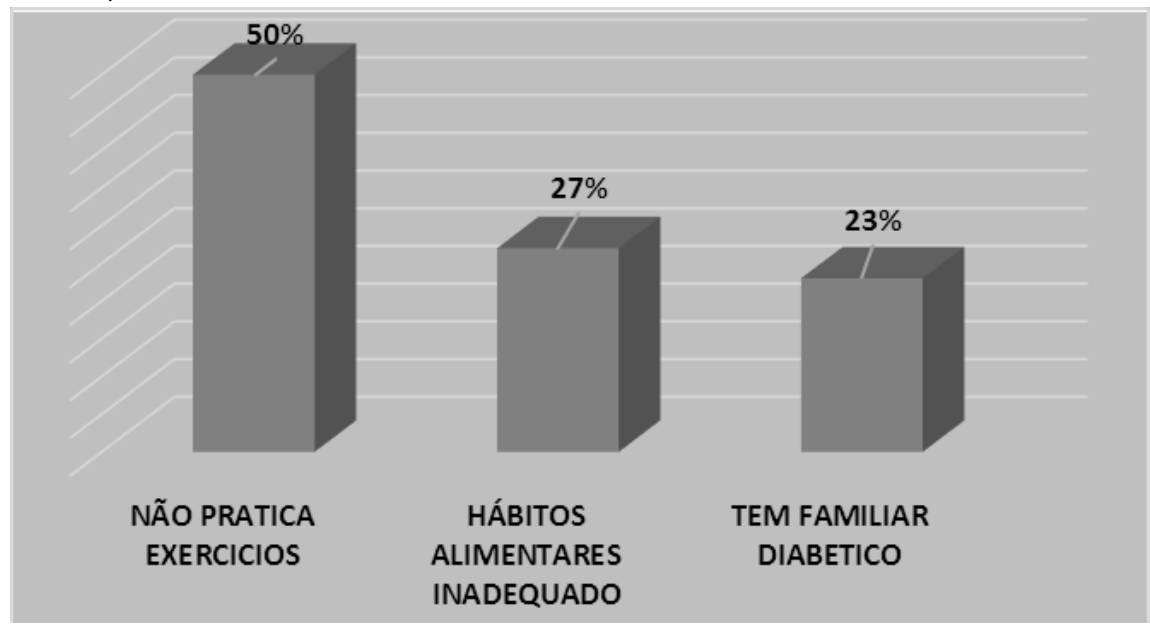

Fonte: Azevedo AP, et al., 2020.

Os resultados quanto ao tabagismo nos dois grupos, também foram significantes, no total $18,0 \%$ relataram que fumavam de 1 a 10 cigarros por dia (Quadro 1). Evidências cientificas mostram que 0 tabagismo é um dos grandes responsável pelos gastos em saúde pública, já que é responsável por um grande número de internação por doenças pulmonares e diminuição de qualidade e duração de vida (BRUNO A, 2014).

Seguindo a avaliação das variáveis encontrada no Quadro 2, percebeu-se que a análise de comparação de gênero mostra uma tendência maior de alteração glicêmica entre as mulheres detectando uma prevalência de risco para doenças cardiovasculares e DM2.

Quadro 2 - Comparação do perfil antropométrica e glicêmica entre homens e mulheres nos dois grupos investigados.

\begin{tabular}{|lccc|}
\hline \multicolumn{1}{c}{ Variáveis } & Comunitários & Serv. da saúde & $\begin{array}{c}\text { Não souberam } \\
\text { responder }\end{array}$ \\
\hline $\begin{array}{l}\text { Homem: que, em algum momento, teve } \\
\text { alteração do seu nível de glicose }\end{array}$ & $7,4 \%$ & $55,6 \%$ & $37 \%$ \\
\hline $\begin{array}{l}\text { Mulher: que, em algum momento, teve alteração } \\
\text { do seu nível de glicose ou diabetes durante a } \\
\text { gravidez, ou filhos com mais de 4 quilos }\end{array}$ & $24,2 \%$ & 48,6 & 27,2 \\
\hline $\begin{array}{l}\text { Medida da cintura: Se homem: mais que } 102 \\
\text { (Risco alto) }\end{array}$ & $40 \%$ & $62,3 \%$ & - ---- \\
\hline Se mulher: mais que 88cm (Risco alto) & $44 \%$ & $38 \%$ & ----- \\
\hline
\end{tabular}

Fonte: Azevedo AP, et al., 2020.

A identificação de fatores de risco tem sido apontada como estratégia fundamental na elaboração das ações que possam, de fato, impactar no processo saúde/doença das pessoas e evitar a ocorrência de agravos. Houve, nos dois grupos investigados, maior ocorrência de participantes do estudo com baixo risco, e risco discretamente moderado (Quadro 3). Estes resultados são menores, porém próximos aos encontrados em estudo realizado em outros locais fechados como empresas salas de aula e grupos específicos (RAMO DE, et al., 2020). 
Uma observação a ser destacada mostra uma participação maior das mulheres na presente pesquisa, o que demonstra um domínio maior das mulheres na comunidade. Isso é um fator a ser analisado já que os indicadores de alto risco são voltados, na sua grande maioria, para o sexo masculino, já que os homens não demonstram um cuidado em relação a sua saúde o que aumenta as complicações de morbidade e mortalidade para esse grupo (ROTH GA, et al., 2015).

Quadro 3 - Resultado da aplicação do questionário Finnish Diabetes Risk Score (segundo a classificação de Findrisck) que mostra o risco de desenvolver DM2 em dez anos.

\begin{tabular}{|lcc|}
\hline \multicolumn{1}{|c}{ Variáveis } & $\begin{array}{c}\text { Moradores } \\
\text { da } \\
\text { Comunidade }\end{array}$ & $\begin{array}{c}\text { Servidores } \\
\text { da saúde }\end{array}$ \\
\hline $\begin{array}{l}\text { < 7 pontos: risco baixo - deve manter as consultas } \\
\text { habituais }\end{array}$ & $8,0 \%$ & $42 \%$ \\
\hline $\begin{array}{l}7 \text { a 14 pontos: pouco elevado - deve modificar o estilo de } \\
\text { vida }\end{array}$ & $49,0 \%$ & $22 \%$ \\
\hline $\begin{array}{l}15 \text { a 20 pontos: risco alto - até três meses para reavaliar } \\
\text { a glicemia com intervenções necessárias }\end{array}$ & $20,0 \%$ & $22 \%$ \\
\hline $\begin{array}{l}>20 \text { pontos: muito alto - Um mês para reavaliar a } \\
\text { glicemia }\end{array}$ & $23,0 \%$ & $37 \%$ \\
\hline
\end{tabular}

Fonte: Azevedo AP, et al., 2020.

Os profissionais da saúde em especial os enfermeiros que atuam na atenção primária, tem um papel fundamental na promoção à saúde. É importante orientar e acompanhar, intervindo nos fatores de risco, orientando a população e implementando ações de prevenção para o DM2 (OLIVEIRA LF, et al., 2016).

Em um estudo realizado por Domingues JG, et al. (2019), se observa que os mesmos evidenciam os hábitos de vida e o estresse da equipe de saúde, principalmente enfermagem, gerados por suas atividades diárias, rotatividade em turnos de trabalho e jornadas noturnas, habito de beber e fumar, não praticar exercícios físicos como fatores que influenciam o desenvolvimento e o agravamento de doenças crônicas não transmissíveis (DCNT), incluindo hipertensão arterial sistêmica (HAS) e diabetes mellitus (DM).

As atividades do enfermeiro são diversificadas, tornando-se um processo complexo, sendo compreendida pelo cuidar, educar e gerenciar. Entretanto, tem-se observado que as práticas de educação em saúde na atenção secundária e terciária têm sido colocadas em segundo plano, já que a atuação da Enfermagem se ocupa de outras tarefas, voltando-se apenas aos serviços assistenciais e de gerência. Acredita-se que o cuidar, associado ao educar, favorece diretamente a assistência em saúde quando há a inclusão do familiar no ambiente hospitalar (AZEVEDO AP, et al., 2018).

Assim, as estratégias de prevenção da ocorrência a partir do reconhecimento dos riscos na comunidade, se tornam essenciais para a garantia da promoção da saúde da população (ZARDO MR, et al., 2015; FORERO AY, et al., 2018). Deste modo, a estratégia significativa para a prevenção é a educação em saúde realizada a partir da conscientização da necessidade de hábitos de vida saudável, e sobre os fatores de risco para o seu desenvolvimento (STRINGHINI S, et al., 2017).

Outros autores também enfatizam a realização de campanhas de conscientização direcionadas não apenas aos profissionais de saúde, mas também à população geral. Essas campanhas, segundo eles, devem ser feitas para que sejam estabelecidos diagnóstico precoce e tratamento adequado (SOUZA LCVF, et al., 2020).

\section{CONCLUSÃO}

Foi evidenciado que $43 \%$ dos participantes da presente pesquisa apresentaram índice muito alto de riscos para desenvolver DM2 nos próximos 10 anos principalmente entre os indivíduos que trabalhavam na instituição de saúde vivenciando a morte e o morrer e que estão em contato direto ou indireto com pacientes portadores de doenças infectocontagiosas expressaram alto estresse em virtude do medo de se contaminar 
com alguma doença contagiosa no ambiente de trabalho e foram os que apresentaram maiores fatores predisponentes para o aparecimento de DM2 diferentemente dos participantes moradores da comunidade que, mesmo tendo restrições financeiras que os impedia de ter uma boa alimentação ou de frequentar a academias, apresentaram maior interesse em manter atividades físicas e com isso apresentaram menos fatores predisponentes.

\section{AGRADECIMENTOS E FINANCIAMENTO}

A coleta de dados foi realizada, tanto na comunidade como entre os servidores da saúde que trabalhavam na Fundação de Medicina Tropical, através de um movimento promovido pela Agência Adventista de Desenvolvimento e Recursos Assistenciais (ADRA) em parceria com o serviço de educação em saúde da Fundação de Medicina Tropical. Os autores agradecem as duas instituições pela oportunidade.

\section{REFERÊNCIAS}

1. ARELLI B, et al. Avaliação da prevalência de fatores de risco para o desenvolvimento de diabetes mellitus tipo 2 em pacientes da Clínica Unesc Saúde, Revista Demetra, 2014; 9(3); 661-680.

2. ARAÚJO LO, et al. Risco para desenvolvimento do diabetes mellitus em usuários da atenção primária a saúde: um estudo transversal. Rev Gaúcha Enferm., 2015; 36(4): 77-83.

3. ANUHARYS P, et al. Intervención educativa en diabéticos tipo 2. Rev Cubana Med Gen Integr [revista en la Internet], 2009; 25(4): 17-29.

4. ANDRADE I, et al. Rastreamento de Diabetes Mellitus Tipo II Na Comunidade De Paripe Realizado No PiscoUnifacs XIII SEPA - Seminário Estudantil de Produção Acadêmica. Revista UNIFACS, 2014.

5. AZEVEDO AP, et al. Educação em saúde para acompanhantes de pacientes internados. Rev enferm UFPE online, 2018; 12(4): 1168-73.

6. BRASIL. Ministério da Saúde. Secretaria de Atenção à Saúde Departamento de Atenção Básica. Acolhimento à demanda espontânea. Cadernos de Atenção Básica, 2013; 28.

7. BRASIL. Ministério da Saúde. Secretaria de Atenção à Saúde, Departamento de Atenção Básica. Estratégias para o cuidado da pessoa com doença crônica: Diabetes Mellitus. Brasília: Ministério da Saúde; Cadernos de Atenção Básica, 2013; 36.

8. BRASIL. Ministério da Saúde | Secretaria de Atenção à Saúde | Departamento de Atenção Básica Estratégias para o Cuidado da Pessoa com Doença Crônica Diabete Mellitus, 2015.

9. BRASIL. Ministério da Saúde. Secretaria de Atenção à Saúde. Departamento de Atenção Básica. Rastreamento. Brasília: Cadernos de Atenção Básica, 2010; 29.

10. BRUNO A, et al. Evaluation of the prevalence of risk factors for development of type 2 diabetes mellitus in patients of the Unesc Clinic. Demetra, 2014;9(3); 661-680.

11. BORGES DB, LACERDA JT. Actions aimed at the Diabetes Mellitus control in Primary Health Care: a proposal of evaluative model. Rio de Janeiro, 2018; 42(116): 162-178.

12. CÂNDIDO JAB, et al. Findrisk: Estratificação Do Risco Para Diabetes Mellitus Na Saúde Coletiva. Rev Bras Promoç Saúde, 2017; 30(3): 1-8.

13. DANTAS R, et al. Utilização do FINDRISC no Rastreio da Diabetes em Utentes Assintomáticos. Serviço de Endocrinologia, Diabetes e Nutrição do Centro Hospitalar do Baixo Vouga. Rev Port Endocrinol Diabetes Metab. 2017; 12(1): 45-51.

14. DOMINGUES JG, et al. Noncommunicable diseases among nursing professionals at a charitable hospital in Southern Brazil. Epidemiol. Serv. Saude, 2019; 28(2): e2018298.

15. FOUAD M, et al. Ácido úrico sérico e sua associação com hipertensão, nefropatia precoce e doença renal crônica em pacientes diabéticos tipo 2. J Bras Nefrol, 2016; 38 (4):403-410.

16. FORERO AY, et al. La alimentación para pacientes con diabetes mellitus de tipo 2 en tres hospitales públicos de Cundinamarca, Colombia. Biomédica, 2018; 38: 355-62.

17. MURRAY CJL. Mortality Collaborators. Global, regional, and national age-sex-specific mortality and life expectancy, 1950-2017: a systematic analysis for the Global Burden of Disease Study 2017. Lancet, 2018; 392: 1684-735.

18. MAZZINI MCR, et al. Diabetes mellitus risk screening of parents of private school students in the city of Jundiaí, São Paulo, Brazil. Rev Assoc Med Bras., 2013; 59:136-42.

19. OLIVEIRA LF, RODRIGUES PA. Circunferência de cintura: protocolos de mensuração e sua aplicabilidade prática. Nutrivisa - Revista de Nutrição e Vigilância em Saúde, 2016.

20. O'DONNELL MJ, et al. Efeitos globais e regionais de fatores de risco potencialmente modificáveis associados a acidente vascular cerebral agudo em 32 países (INTERSTROKE): um estudo de caso-controle. Lanceta, 2016; 388: 761-775.

21. RAMO DE, et al. Multiple Health Risk Behaviors in Young Adult Smokers: Stages of Change and Stability over Time. Ann Behav Med., 2020;54(2):75-86. 
22. ROTH GA, et al. Carga global, regional e nacional de doenças cardiovasculares por 10 causas, 1990 a 2015 . J Am Coll Cardiol. 2017; 70:1-25.

23. SKALAMERA J, HUMMER RA. Educational attainment and the clustering of health-related behavior among U.S. young adults. Prev Med., 2016; 84: 83-89.

24. STRINGHINI S, et al. Socioeconomic status and the $25 \times 25$ risk factors as determinants of premature mortality: a multicohort study and meta-analysis of 1.7 million men and women [published correction appears in Lancet, 2017; 389(10075): 1194.

25. SOCIEDADE BRASILEIRA DE DIABETES. Departamento de Epidemiologia, Economia e Saúde Pública. Dados Epidemiológicos do diabetes Mellitus no Brasil. SBD 2018 - 2019.

26. SOUZA LCVF, et al. Diabetic ketoacidosis as the initial presentation of type 1 diabetes in children and adolescents: epidemiological study in southern Brazil. Rev Paul Pediatr., 2020; 38:e2018204.

27. ZARDO MR, et al. Rastreamento de fatores de risco para diabetes tipo $2 \mathrm{em}$ trabalhadores de uma indústria da cidade de Concórdia - SC. Perspectiva, Erechim., 2015; 39(145): 85-95. 